Notfall Rettungsmed 2010 $13: 753-753$

DOI 10.1007/s10049-010-1304-0

Online publiziert: 25. November 2010

(c) Springer-Verlag 2010

\author{
H.-R. Arntz ${ }^{1} \cdot$ R. Somasundaram ${ }^{2}$ \\ ${ }^{1}$ Medizinische Klinik II, Kardiologie und Pulmonologie, \\ Charité Universitätsmedizin Berlin, Campus Benjamin Franklin, Berlin \\ ${ }^{2}$ Interdisziplinäre Rettungsstelle und Aufnahmestation, \\ Charité Universitätsmedizin Berlin, Campus Benjamin Franklin, Berlin
}

\title{
Innerklinische Notfälle
}

\section{Sind wir gut vorbereitet?}

Hand aufs Herz: Wer hat nicht schon einmal eine Reanimation im eigenen Hause erlebt, bei welcher in der späteren Aufarbeitung nicht der Gedanke kam, ob man die klinische Entwicklung dieses Patienten hätte nicht schon früher erkennen können oder müssen. Wäre die Reanimation nicht potenziell vermeidbar gewesen? Sind die Überbrückungsmaßnahmen, wie z. B. der Basic Life Support, bis zum Eintreffen des Reanimationsteams wirklich effektiv gewesen? Warum kannten sich die ärztlichen Kollegen und die Pflegekräfte der Station so schlecht mit der Notfallausstattung (Defibrillator, Medikamente, Absaugung, etc.) auf Station aus? Was für Konsequenzen sind aus dem jeweiligen Fall zu ziehen bzw. wie lassen sich künftig solche Abläufe verbessern?

Das Management innerklinischer Notfälle ist kein isoliertes Problem nur weniger Kliniken oder Länder. Dies ist auch daran erkennbar, dass in den gerade veröffentlichten Leitlinien des European Resuscitation Council (ERC) der in der Klinik eintretende Herz-Kreislauf-Stillstand und sein Management ebenfalls thematisiert werden. Im Gegensatz zum präklinischen Auftreten ist der innerklinische Herz-Kreislauf-Stillstand allerdings oft vorhersehbar bzw. kündigt sich z. B. durch eine Hypoxämie und/oder eine Hypotension an, die nicht frühzeitig registriert wurde, d. h. möglicherweise wäre eine Reanimation vermeidbar gewesen. Vor diesem Hintergrund empfehlen die aktuellen ERC-Leitlinien, dem Personal Kriterien an die Hand zu geben, sich anbahnende
Komplikationen im Vorfeld zu erkennen und dann ggf. hierfür eingerichtete Präventionsteams der Klinik zu alarmieren. Auch wenn die Effektivität solcher Frühwarn- und Interventionssysteme in Bezug auf das Gesamtüberleben noch nicht eindeutig gezeigt worden ist, besteht international doch zunehmend ein Konsens, solche Systeme zu etablieren. Denn letztendlich kann ein solches erst nach Etablierung ausreichend gut validiert werden.

\section{(7) Der innerklinische Herz-Kreislauf-Stillstand ist oft vorhersehbar oder kündigt sich an}

Das vorliegende Leitthemenheft will die Leser von Notfall + Rettungsmedizin mit dem aktuellen Stand der Diskussion zum Management von innerklinischen Notfällen vertraut machen. Breckwoldt et al. gehen neben der Organisation von Materialvorhaltungen insbesondere auch auf Ausbildung und Training von Mitarbeitern ein. Dies ist ein sicher ganz entscheidender Punkt im Erkennen und Behandeln von Notfällen. Fischer et al. beleuchten die tatsächlichen wissenschaftlichen Evidenzen, die für die Etablierung von Frühwarnsystemen und Interventionsteams sprechen. Leidel und Kanz diskutieren die Besonderheiten von chirurgischen Bereichen in Bezug auf innerklinische Notfälle und gehen hier ebenfalls auf die Etablierung von standardisierten Präventionssystemen ein. In all diesen Bereichen stellen aber auch neurologische
Notfälle und ihre Erkennung im Vorfeld für Kollegen aus der Notfallmedizin immer eine besondere Herausforderung dar, da auch hier ein zu spätes Reagieren oft fatale Folgen - selbst nach Überleben - hat. Der Beitrag von Pfefferkorn gibt hier eine ausgezeichnete und konzise Übersicht der neurologischen Notfälle, ihrer Diagnose und Therapie, wie sie auch für den NichtNeurologen sehr wichtig sind.

Wir wünschen uns, dass Sie als Leser mit diesen Beiträgen vielleicht auch eine weitere Argumentationsgrundlage für die Verbesserung der Prozessabläufe bei innerklinischen Notfällen und damit auch der Versorgungsqualität Ihrer Klinik erhalten.

Mit den besten Wünschen

Ihre

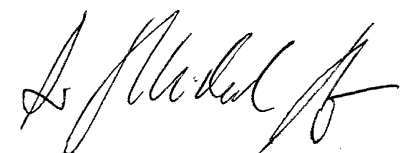

Prof. Dr. Hans-Richard Arntz

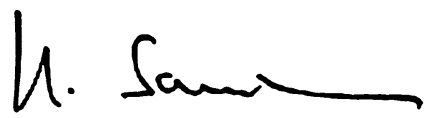

Prof. Dr. Rajan Somasundaram

\section{Korrespondenzadresse}

Prof. Dr. R. Somasundaram

Charité Universitätsmedizin Berlin, Campus Benjamin Franklin, Interdisziplinäre Rettungsstelle und Aufnahmestation 12200 Berlin

Rajan.Somasundaram@charite.de 